\title{
Performance Analysis of Relay Enhanced LTE-Advanced Network
}

\author{
Muhammad Aslam \\ ICT, UET Peshawar \\ Pakistan
}

\author{
Aftab Hussain, PhD \\ Associate Professor, PSIT \\ Pakistan
}

\author{
Nadeem ullah Khan \\ PTCL, Islamabad \\ Pakistan
}

\begin{abstract}
Long Term Evaluation (LTE) Advanced networks are popular due to very high data rates with ultra-wide bandwidth spectrum, and broad coverage and are currently adopted as 4G standard. In spite of vast benefits of LTE Advanced, the propagation loss occurs due to low signal-to-noise-ratio (SNR) and results in low coverage and channel capacity at the cell boundaries. To overcome these problems, cooperative communication network is used by deploying relay nodes. In this paper, we conduct the performance analysis of LTE Advanced relay system on WINNER channel model with various communication scenarios. The simulations environment for the LTE Advanced network and WINNER channel models is created using Matlab. For easiness, only one base station and user equipment are used in the simulation setup. We observe the effect on LTE performance using one or two relay nodes in the LTE network. We conduct the experiments for four different environments and six scenarios. We analyze the performance using symbol-error-rate for different SNR. The results show that LTE Advanced network using relay nodes performs much better than other environments. It also provides least latencies, even wider coverage, and higher data rate with minimum outage.
\end{abstract}

\section{General Terms}

Algorithms.

\section{Keywords}

Long Term Evolution, Relay Access Network, Relay Node, LTE-Advanced.

\section{INTRODUCTION}

In the mobile communication world, Long Term Evolution Advanced (LTE-A) [1] is currently the main network that is being worked on. The main purpose of its development was to meet the requirements set by the International Telecommunication Union (ITU). The LTE-A is equally compatible with the previous releases of LTE network and can support sharing of frequency bands with earlier releases. The main advantage of LTE-A network is that it can adopt any advanced network topology which may include low power nodes, and other heterogeneous network items including relay nodes [2]. LTE Advanced network also equipped with multi carriers achieving ultra-wide bandwidth up to $100 \mathrm{MHZ}$ of spectrum supporting to handle very high data rate which makes LTE Advanced a suitable standard for $4 \mathrm{G}$ in the current era. To meet the different wireless communication challenges from short range to wide range communication and to adopt different communication scenarios, a ubiquitous radio access system is required. An efficient radio system is very crucial to evaluate different transceiver constraints including modulation, coding, link adaptation, and throughput. With advancement in technology, the propagation loss is still a problem. Due to the low signal- to-noise ratio (SNR), coverage and capacity remain low at the cell borders. To overcome this problem, Relay Nodes (RN) are used in the network [3-4]. The RN near the cell edges enhances the capacity and coverage The $\mathrm{RN}$ can be considered as a cooperative communication in which an RN transfers the information from a local evolved Node B (eNB) to neighboring User Equipment (UE) [5-6]. Therefore, an RN shifts the service and signal from one eNB to UE which increases the system throughput and enhances the SNR. 5.4 Comparison with the existing technique

In the literature, Abdullah et al. in [7] worked for the enhancement of LTE Advanced relay deployment by performing tests to observe the performance of the LTE Network through relay deployment. The simulated network was represented by a regular hexagonal cellular layout with 19 tri-sectored sites. The RNs admit regular outdoor deployment at the sector border. The performance was compared in urban and suburban scenarios. In [7], network gain was increased by increasing the number of RNs while in our work, network performance is analyzed by adding one RN between UE and BS and it increases the SNR and gain of the systems.

In this work, we aim to evaluate the performance of LTE Advanced relay system. For simulation of the LTE Advanced network, only WINNER model has been utilized since it is the only model available open source [8]. The simulation is carried out in Matlab. No actual device or environment is used so results produced will be more theoretical rather than practical.

The rest of the paper is as follows. In Section 2, the proposed algorithm and simulation parameters are described. In Section 3, different wireless network scenarios, their implementations, and interpretation of their results will be discussed. Finally, the paper is concluded along with future recommendations in Section 4.

\section{PROPOSED ALGORITHM}

The advantage of LTE Advanced network is the use of relays in $4 \mathrm{G}$ network that help to increase the network capacity and data rate. In this paper, we use the WINNER channel model as a base model for simulation. WINNER model works on physical layer. The main components involved in the simulation are scenarios, base station, relay nodes, and user equipment. The description of these components is provided in the following sections.

\subsection{Scenarios}

WINNER channel model has been used in the simulator which deals with the environment conditions and scenarios to implement the radio technology. WINNER channel model is an effective model among the models to specify the channel features of indoor and outdoor environments. The focus of 
this environment scenario is to increase the network capacity. WINNER channel model has fourteen different scenarios [9]. These scenarios provide multiple environment situations however they do not cover all environment conditions such as hills and mountains. Also, heights of antennas do not ensure all possible reasonable values. The scenarios considered are generally for urban areas where the environment has been divided into two groups.

\subsection{Base Station}

In this simulator, only one Base Station (BS) is considered. The Base Station comprises three-sectored Omni-directional antennas. It means antenna pattern used for the BS is 3sectored. Other antenna patterns include 1-sector and 2-sector which can also be considered.

\subsection{Relay Nodes (RNs)}

The RNs considered in the simulator are simple amplify-andforward RN. The node is best suited for a low or limited noise environment. It is assumed that relay has no latency and there is no scheduling. The model for RN includes one UE on the testing side and one BS in transmission.

\subsection{User Equipment (UE)}

The UE is assumed to be static or non-mobile in this case. UE is basically a single direction antenna which has no impact on the model. The model considered is independent of the UE.

Table 1. List of simulation parameters used

\begin{tabular}{|l|l|l|l|}
\hline Parameter & Value & Parameter & Value \\
\hline $\begin{array}{l}\text { Transmitter } \\
\text { power }\end{array}$ & 1 watt & $\begin{array}{l}\text { Channel } \\
\text { bandwidth }\end{array}$ & $20 \mathrm{MHz}$ \\
\hline $\begin{array}{l}\text { Carrier } \\
\text { frequency }\end{array}$ & $2 \mathrm{GHz}$ & BS sector & 1 \\
\hline $\begin{array}{l}\text { Number of } \\
\text { cells }\end{array}$ & 1 & BS antenna & 1 \\
\hline $\begin{array}{l}\text { Channel } \\
\text { Model }\end{array}$ & WINNER II & Antenna gain & $14 \mathrm{dBi}$ \\
\hline $\begin{array}{l}\text { Downlink } \\
\text { buffer }\end{array}$ & Full & BS noise figure & $5 \mathrm{~dB}$ \\
\hline BS height & $32 \mathrm{~m}$ & RN height & $25 \mathrm{~m}$ \\
\hline $\begin{array}{l}\text { Number of } \\
\text { UE }\end{array}$ & 1 & No. of relays & 1 \\
\hline UE antenna & 2 & Relay antennas & 1 \\
\hline $\begin{array}{l}\text { Relay } \\
\text { transmit } \\
\text { power }\end{array}$ & $1 \mathrm{~W}$ & $\begin{array}{l}\text { Relay } \\
\text { antenna gain }\end{array}$ & $9 \mathrm{dBi}$ \\
\hline $\begin{array}{l}\text { Relay node } \\
\text { noise } \\
\text { Figure }\end{array}$ & $7 \mathrm{~dB}$ & $\begin{array}{l}\text { User equipment } \\
\text { height }\end{array}$ & $1.5 \mathrm{~m}$ \\
\hline $\begin{array}{l}\text { UE noise } \\
\text { Figure }\end{array}$ & $7 \mathrm{~dB}$ & & \\
\hline
\end{tabular}

For simulation, we use regular cellular cell shape i.e., hexagonal cell structure. The cell includes RN, one UE, and one BS. The height of the RN is in between the heights of the BS and UE. The simulation is only done for the downlink path. The simulation parameters are listed in Table 1.

To deploy the RN in the cell and to enhance the network capacity and performance, it is ensured to setup a fixed distance between BS, UE, and RN. One such arrangement is shown in Figure 1a, where distance between UE and BS is set as $1000 \mathrm{~m}$ and relay is installed just in the middle thus making the distance of relay node with BS and UE as $500 \mathrm{~m}$. Network environment with 2 relay nodes has been shown in Figure $1 \mathrm{~b}$. Here, BS and UE are placed at same fixed distance of $1000 \mathrm{~m}$ however; two relays are placed in such a way that they both maintain an equal distance with both BS and UE. In such case, they are setup at a distance of $559 \mathrm{~m}$ from both BS and UE. A generalized algorithm for the simulation is developed using WINNER model. The flowchart of the algorithm is shown in Figure 5. The algorithm starts with the definition of orientation of BSs and UEs along with antenna arrays for noncooperative, co-operative, and without relay network. BSs and UE are oriented in such a way that BS and UE in relay node must be in same order to perform as a single entity. Second block deals with the WINNER model scenarios and parameters related to those scenarios. Using the orientation of BS and UE and the selected scenario, design parameters are set. Third block affirms the positions of all BSs, UEs, and RNs in the designated layout in previous step. All positions with heights are oriented in three dimensional Cartesian coordinate system. Then pairing matrix is defined to deal with defining relay links between UEs and BSs.

After defining the necessary parameters and configuration required for the simulation, the links for WINNER model are generated. Using MATLAB code, simulation links are generated. The subsequent steps in the algorithm design are the OFDM simulator block. This block is responsible for channel filtering using the path powers from previous steps. There are different OFDM blocks for different environments involved. For the non-cooperative environment, OFDM block works in two steps since the data is transmitted in two steps i.e., from $\mathrm{BS}$ to $\mathrm{RN}$ and $\mathrm{RN}$ to UE. In the co-operative environment, the receiver receives the data in two separate ways i.e., without relay link (direct link) and through access link and relay link. ODFM block for this environment adds the data from both links at transmission and relay-to-access link at receiving.

Next block deals with the calculations of SER. The SER is calculated for three environments separately. The SER is calculated each time WINNER model is used to generate path powers of combined paths and links. The process from generation WINNER links to the calculation of SER is performed in 500 iterations. In each iteration, SER calculated is added to previously calculated SER for the respective environment. At the end, the average is computed. The average SER is plotted against SNR of each environment.

\section{EXPERIMENTAL RESULTS AND DISCUSSIONS}

In this paper, we used six wireless communication scenarios of WINNER channel model i.e., indoor to outdoor, model inner-city micro-cell, bad inner-city micro cell, semi-urban, model inner-city macro cell, and bad inner-city macro cell scenarios.

The distance between base station and UE is fixed at $1000 \mathrm{~m}$. The reason for keeping this distance in the proposed model is that it is an effective model for LOS probability [10] at a small distance. Also, it does not go to zero coverage at a cell boundary. All the scenarios taken into consideration have three links named Access Link, Direct Link, and Relay Link. Based on these, each scenario has three environments described below. 


\subsection{Without relay environment}

In this environment, no Relay Node is utilized. There is a Direct Link between BS and UE.

\subsection{Non-cooperative Environment}

This type of environment includes an RN. The signal is transmitted from BS to RN then from RN to UE. Both Relay Link and Access Links are used.

\subsection{Co-operative Environment}

This type of environment transmits the signal from BS to UE via two links. First is the Direct Link between BS and UE. Second is the use of a Relay Link and the Access Link. Cooperative environment makes use of the both environments i.e. without relay and non-co-operative. UE receives the signal from both links.

\subsection{Co-operative Environment with Two Relays}

This type of environment includes two RNs. The signal from BS to UE is transmitted in three paths. The first path is the direct path between BS and UE using the Direct Link. The second path is through $\mathrm{RN}_{1}$. The signal travels from $\mathrm{BS}$ to $\mathrm{RN}_{1}$ and then from $\mathrm{RN}_{1}$ to UE. The third path is through $\mathrm{RN}_{2}$. Signal propagated from $\mathrm{BS}$ to $\mathrm{UE}$ via $\mathrm{RN}_{2}$. Figure 1 shows simulation setups for both non-co-operative and co-operative environments.

\subsection{Indoor to Outdoor Scenario}

In this scenario, mobile station (MS) height is taken as 1-2 meters while the BS is fixed at height of 2-2.25 meters. The height of floor is added to the height of BS as well. Simulation is performed for floor 1-3 where floor 1 is the ground floor. Path loss (PL) for this scenario is calculated as

\section{$P L=$}

$$
\begin{aligned}
& 10 \log _{10}(d)+9.45-17.3 \log _{10}\left(h_{B S}\right)-17.3 \log _{10}\left(h_{U E}\right)+ \\
& 27 \log _{10}\left(f_{c} / 5\right) x_{d}+14(15(1-\cos \theta))^{2}+0.5 d
\end{aligned}
$$

where $d$ is the distance between $T_{X}$ and $R_{X}, h_{B S}$ is the height of $B S, h_{U E}$ is height of UE, $f_{c}$ is carrier frequency and $\theta$ is aperture angle of antenna.

The SNR vs. SER results for indoor to outdoor scenario are shown in Figure 3a. From the simulation results, we observe that without relay environment has much high symbol error rate at higher SNR which is not good. SER in co-operative environment curve is very low as compared to other environments. The curve showing the co-operative network with two relays has the best result. It has the least SER on higher SNR which implies that environment using relays is better than the other environments.

\subsection{Model Inner-city Micro-cell Scenario}

The urban micro cell is a grid of streets where one street having full coverage is considered as 'Main Street' in which line of sight (LOS) is good from all locations to BS. LOS may be lost for a while due to traffic. Street running parallel to main streets are called 'parallel streets' while streets intersecting the main street are called vertical streets. The signal propagates from building corners and from in between. path loss for this scenario is given below.

$$
\begin{aligned}
& P L= \\
& 10 \log _{10}(d)+9.45-17.3 \log _{10}\left(h_{B S}\right)-17.3 \log _{10}\left(h_{U E}\right)+ \\
& 2.7 \log _{10}\left(f_{c} / 5\right)
\end{aligned}
$$

Figure 3b shows Model Inner-city Micro-cell scenario plots for SNR vs. SER. From the results, it is evident that up to 8 $\mathrm{dB}$ all the environment exhibits the same results almost, but on higher SNR, Co-operative relays environments performs better.

\subsection{Bad Inner-city Micro Cell Scenario}

In this scenario, the layout of the cell is similar to the model micro cell in an urban area; however, propagation characteristics are worse. The signals are not properly received at every location. The multipath energy from reserved objects can be received at some locations only. Such propagation happens when there are water bodies, parks or squares in the cell. The path loss is calculated as follows:

$P L=13.9 \log _{10}(d)+64.4+20 \log _{10}\left(f_{c} / 5\right)$

Figure 3c shows SNR vs. SER graph results for Bad Micro cell in the urban area. The results show that without relay environment has the worst results having highest SER. Up to $8 \mathrm{~dB}$, both co-operative networks have the same SER, with the increase in SNR; co-operative network with two relays performs better than a co-operative network with one relay.

\subsection{Semi-urban Macro-Cell Scenario}

In this scenario, BSs are located on the roof tops while MSs are on streets. It is assumed that buildings in the residential area and they occupy single or double floors only. Parks in the area make the cell more open. The streets are not assumed to be in complex intersection. Path loss equation is given as:

$P L=40 \log _{10}(d)+11.65-16.2 \log _{10}\left(h_{U E}\right)+$ $3.8 \log _{10}\left(f_{c} / 5\right)$

Figure 3d shows semi-urban macro-cell scenario plots of SNR vs. SER. The results show that without relay environment has the highest SER on higher SNR while co-operative environment with two relays has the least SER .Comparing with other environments, a two-relay environment is the best in the environments.

\subsection{Model Inner-city Macro Cell Scenario}

In this scenario, MSs are usually located at street level whereas BSs are installed at the top of buildings comprising of four floors. Propagation characteristics may face a congested or non-obstructed line of sight (LOS). Height and density of the buildings in the model urban macro cell are homogeneous. Path loss equation is given as.

$P L=$

$10 \log _{10}(d)+13.47-14 \log _{10}\left(h_{B S}\right)-14 \log _{10}\left(h_{U E}\right)+$ $6 \log _{10}\left(f_{c} / 5\right)$

Figure 3e shows Model Inner-city Macro-cell scenario results of SNR vs. SER. The results show that above $20 \mathrm{~dB}$, the performance of the network with two relays is better than all other environments since it has the least symbol error rate

\subsection{Bad Inner-city Macro Cell Scenario}

A bad macro cell in the urban area geometrically contains the inhomogeneous building heights and densities which lead to propagation imperatives. BSs are usually mounted on the buildings having above average heights still the propagation is disturbed as there may be high rise buildings in its coverage. Path loss for this type of cell is given as:

$P L=$

$44.9-6.55 \log _{10}\left(h_{B S}\right) \log _{10}(d) 34.43+5.83 \log _{10}\left(h_{B S}\right)+$ $23 \log _{10}\left(f_{c} / 5\right)$ 
The results in Figure $3 \mathrm{f}$ show that the performance of the network with two relays in this scenario is better than all other environments included since it has the least symbol error rate.

From the simulation results of different scenarios, it is inferred that co-operative environment is better than other non-co-operative environment and without relay environment. In co-operative environments, the network containing two relays is even better than the network with one relay. The reason for this better performance is that in the environment involving two relays, UE receives the power from three links (Direct Link and two Access Links). Received power at UE from all links is added up and hence enhances the SNR and reduces the SER. In these, simulations, interference factor, however, taken not been considered. It can be stated clearly that deployment relays or relay combination in LTE advanced network enhances the system coverage and SNR and can be effectively implemented in comparison to other existing techniques.

\section{CONCLUSION}

In this paper, we observed the effects of deploying RNs in the existing structure of LTE network on cell boundary using WINNER II channel model. The performance of the network was evaluated by introducing an RN between BS and UE and simulations were carried out for four different environments and six scenarios. The results showed that relay network performs much better than other environments. It has been shown that LTE Advanced network performs much better than LTE with the help of RNs installed in the existing network. The simulation results showed that received power added up to the co-operative network via different links helps to improve SNR thus reducing the SER. The performance of the non-cooperative network and without relay network is lower since powers are not added up to the network. Cooperative network with relay presented even better performance if another relay is added for support.

\section{ACKNOWLEDGMENTS}

We would like to thank the reviewer for their valuable comments.

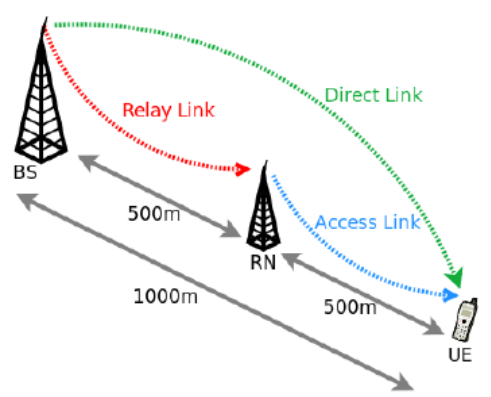

(a)

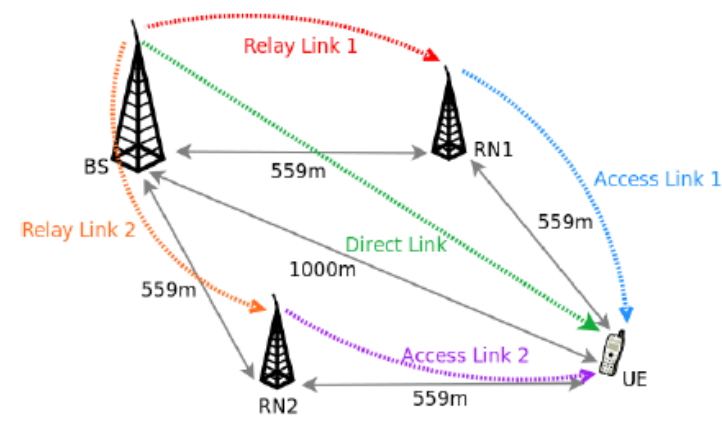

(b)

Figure 1. Simulation setups for (a) Non-cooperative environment (b) Cooperative environment

\section{REFERENCES}

[1] E. Dahlman, S. Parkvall, and J. Skold, 4G: LTE/LTEadvanced for mobile broadband. Academic press, 2013.

[2] Motorola, "Long Term Evolution: A Technical overview," 2007.

[3] Tommaso Beniero, Simone Redana, Jyri Hämäläinen, Bernhard Raaf; Effect of Relaying on Coverage in 3GPP LTE-Advanced; IEEE 69th Vehicular Technology Conference, VTC Spring 2009, Barcelona.

[4] Yang Yang, Honglin $\mathrm{Hu}$ and Jing $\mathrm{Xu}$, Guoqiang Mao; Relay Technologies for WiMAX and LTE-Advanced Mobile Systems; IEEE Communications Magazine, October 2009.

[5] T. Beniero, S. Redana, J. Hamalainen, and B. Raaf, "Effect of relaying on coverage in 3GPP LTEadvanced," in Vehicular Technology Conference, 2009. VTC Spring 2009. IEEE 69th, 2009, pp. 1-5.

[6] Y. Yang, H. Hu, J. Xu, and G. Mao, "Relay technologies for WiMAX and LTE-advanced mobile systems," IEEE Commun. Mag., vol. 47, no. 10, 2009.

[7] Saleh, Abdallah Bou, Ömer Bulakci, Simone Redana, Bernhard Raaf, and Jyri Hämäläinen. "Enhancing LTEadvanced relay deployments via biasing in cell selection and handover decision." In Personal Indoor and Mobile Radio Communications (PIMRC), 2010 IEEE 21st International Symposium on, pp. 2277-2281. IEEE, 2010.

[8] D. S. Baum et al., "Final report on link level and system level channel models," Win. Deliv. D, vol. 5, 2005.

[9] Thomas Wirth, V. Venkatkumar, Thomas Haustein, Egon Schulz, Rüdiger Halfmann; LTE- Advanced Relaying for Outdoor Range Extension; IEEE 70th Vehicular Technology Conference Fall (VTC 2009-Fall), 2009, Anchorage, AK

[10] "3rd Generation Partnership Project; Technical specification group radio access network; 3GPP TR25.996, Spatial channel model for MIMO simulations(release 6), V6.1.0.”. 


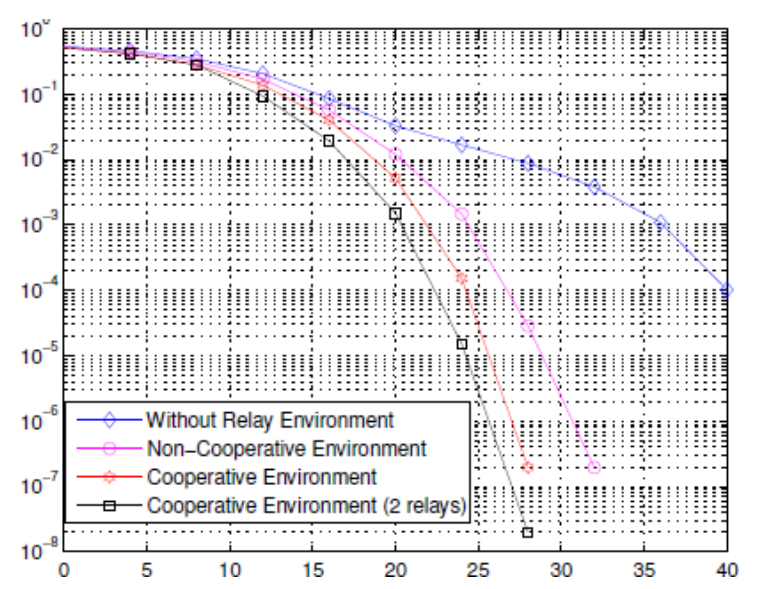

(a)

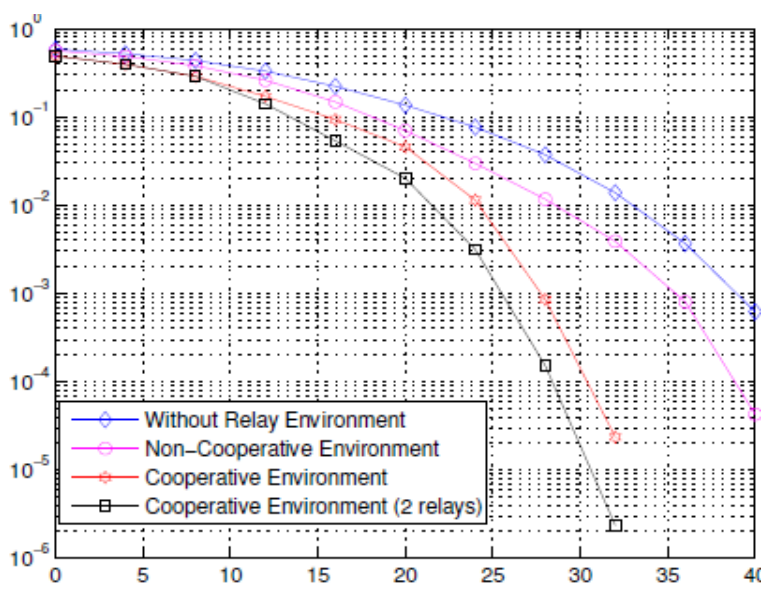

(c)

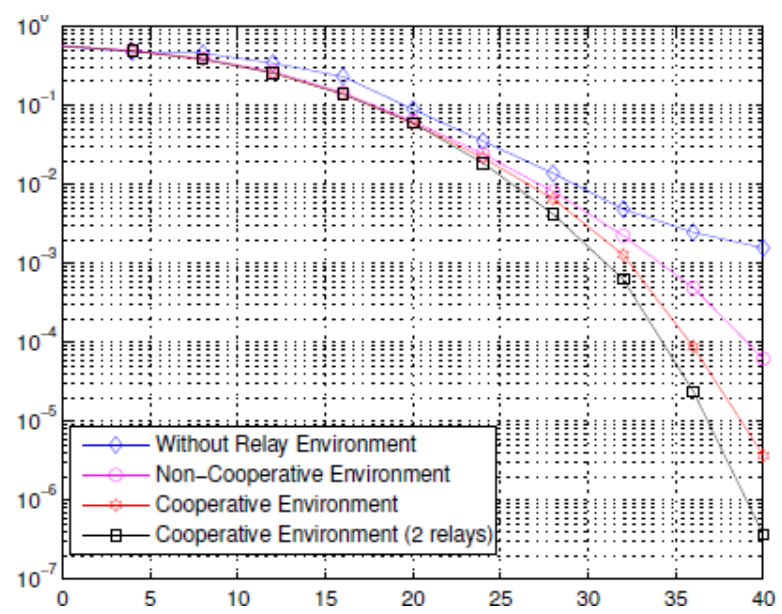

(e)

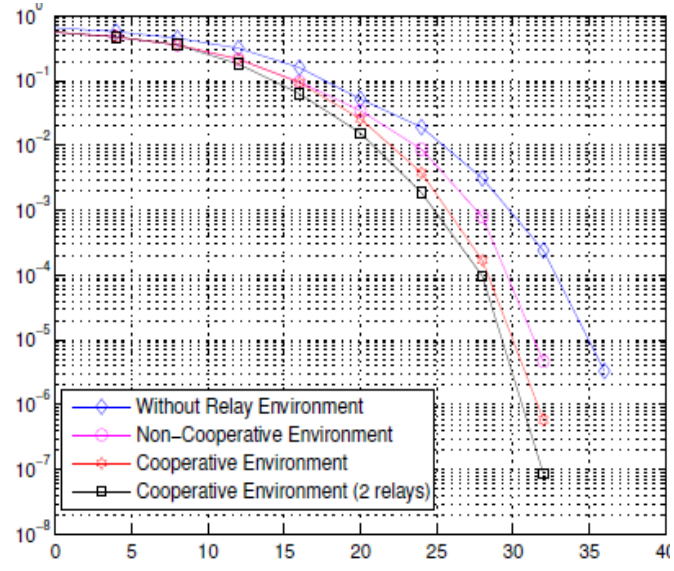

(b)

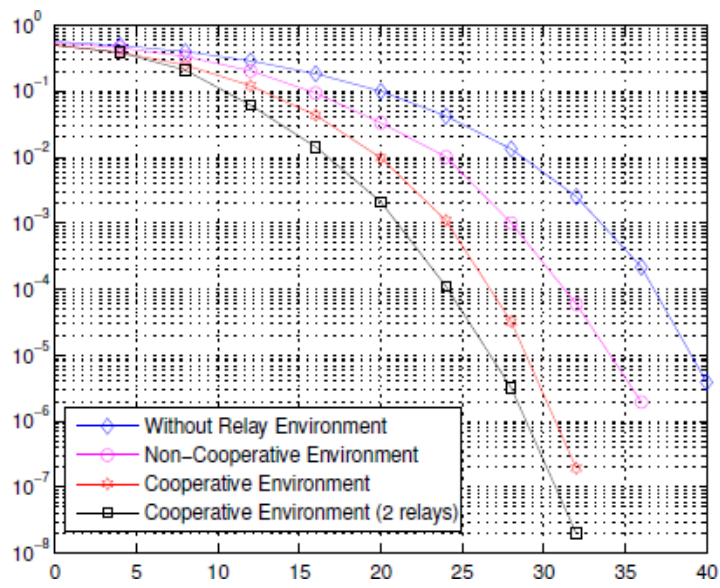

(d)

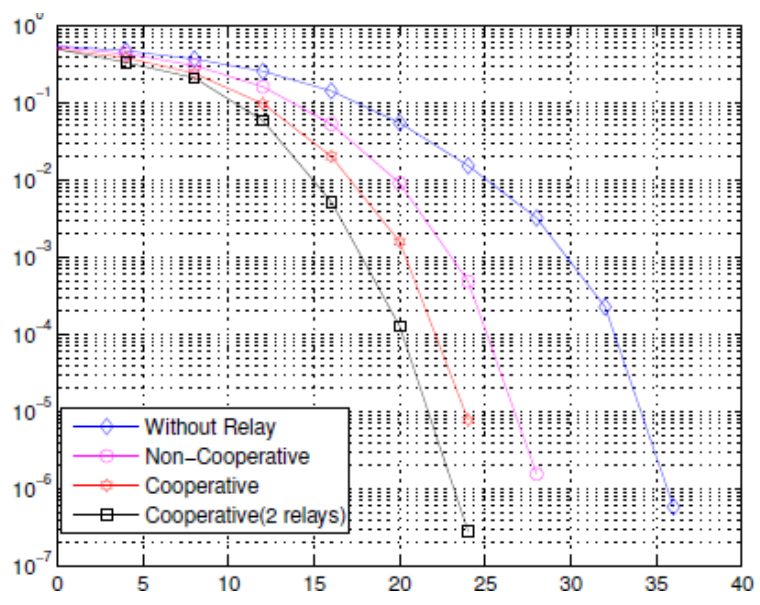

(f)

Figure 3. SNR versus SER for six different environments (a) Indoor to Outdoor (b) Inner-City Micro-Cell (c) Bad Micro Cell in the urban area (d) Semi-urban Macro Cell (e) Urban Macro cell (f) Bad Inner-city Macro Cell 


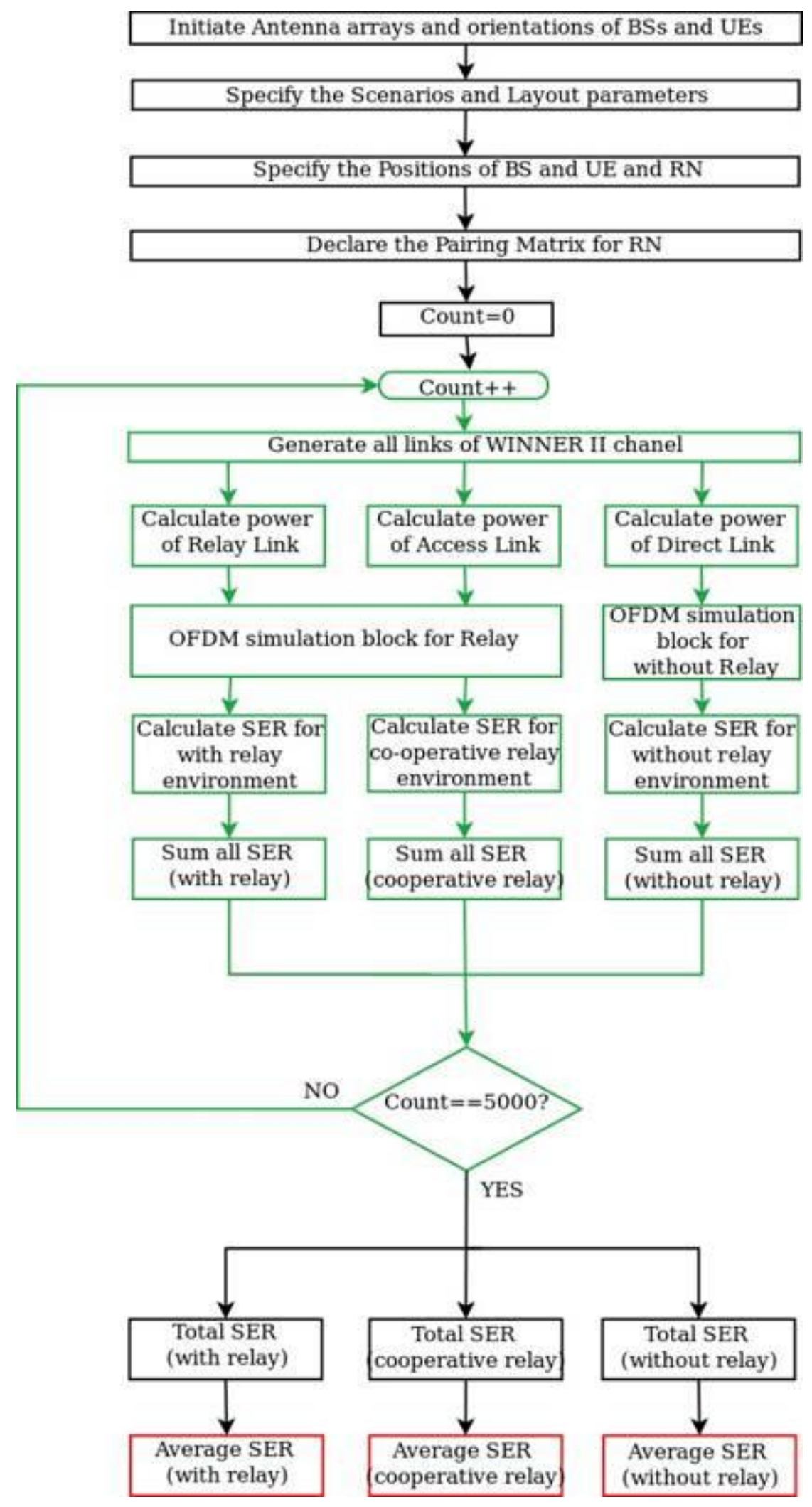

Figure 2. Flowchart of the proposed algorithm 